\title{
Activities of Serum Adenosine Deaminase and its Isoenzymes in Patients with Systemic Lupus Erythematosus, Rheumatoid Arthritis, Ankylosing Spondylitis and Myasthenia Gravis Die Aktivitäten der Adenosin-Desaminase im Serum sowie deren Isoenzyme bei Patienten mit systemischem Lupus erythematodes, rheumatoider Arthritis, Spondylitis ankylosans und Myasthenia gravis
}

Authors

Gao Zhao-wei, Guan-hua Zhao, Rui-cheng Li, Hui-ping Wang, Chong Liu, Hui-zhong Zhang, Ke Dong

Affiliations

Fourth Military Medical University, Department of Clinical Diagnosis, Tangdu Hospital, Xi'an, China

Schlüsselwörter

ADA-Aktivität, Isoenzym, Autoimmunerkrankung, Diagnose

Key words

ADA activity, isoenzyme, autoimmune disease, diagnosis

Bibliography

DOI https://doi.org/0.1055/a-1024-3495

Online-Publikation: 21.11.2019

Akt Rheumatol 2020; 45: 348-355

(c) Georg Thieme Verlag KG Stuttgart · New York ISSN 0341-051X

Correspondence

Dr. Ke Dong

Department of Clinical Diagnosis

Tangdu Hospital

Fourth Military Medical University

Xi'an

China

tdjyk3@fmmu.edu.cn

\section{ABSTRACT}

Objective The aim of this study was to evaluate the changes and diagnostic value of serum ADA activity in autoimmune diseases, including systemic lupus erythematosus (SLE), rheumatoid arthritis (RA), ankylosing spondylitis (AS), and myasthenia gravis (MG).

Methods Serum ADA activity, including total ADA (tADA) and its isoenzymes (ADA1 and ADA2), was determined in patients with different autoimmune diseases (144 RA, 114 SLE, 55 AS, $68 \mathrm{MG}$ ). The changes in serum ADA activity in patients were analysed. A receiver operating characteristic (ROC) curve ana- lysis was applied to evaluate the diagnostic performance of serum ADA activity.

Results Compared with healthy controls, the serum tADA activity in SLE patients was significantly increased $(p<0.001)$, while the serum tADA activity in patients with RA, AS and MG did not change $(p>0.05)$. The ROC analysis showed that the optimal cut-off value of serum tADA activity for SLE diagnosis was $10.5 \mathrm{U} / \mathrm{L}$ (79.8\% specificity and $74.6 \%$ sensitivity; likelihood ratio (LR): 3.693; $\mathrm{p}<0.001)$. Moreover, our results showed that there were no significant changes of ADA1 and ADA2 activity in RA, AS and MG patients, while the serum ADA2 activity was significantly increased in SLE patients. The ROC analysis showed that ADA2 activity could be used in diagnosing SLE with $75.4 \%$ specificity and $78.1 \%$ sensitivity (LR: 3.175$)$. Based on the ROC curve analysis, serum tADA activity (79.8\% specificity and $74.6 \%$ sensitivity; likelihood ratio (LR): 3.693 ) and ADA2 activity (75.4\% specificity and $78.1 \%$ sensitivity; LR: 3.175) are unlikely to be used in diagnosing SLE. Furthermore, there was a positive correlation between tADA activity and SLE disease activity $(r=0.303, p=0.010)$. Notably, serum tADA activity in SLE patients with arthritis was higher than in patients without arthritis $(p=0.005)$, which suggests that tADA activity might be related to lupus arthritis.

Conclusion These findings suggest that serum tADA and ADA2 activity might play an important role in SLE progression.

\section{ZUSAMMENFASSUNG}

Ziel Ziel dieser Studie war es, die Veränderungen sowie den diagnostischen Wert der ADA-Aktivität im Serum bei Autoimmunerkrankungen, einschließlich des systemischen Lupus erythematodes (SLE), der rheumatoiden Arthritis (RA), der Spondylitis ankylosans (AS) sowie der Myasthenia gravis (MG) zu beurteilen.

Methoden Die ADA-Aktivitäten im Serum, einschließlich Gesamt-ADA (tADA) sowie ihrer Isoenzyme (ADA1 und ADA2) wurden bei Patienten mit verschiedenen Autoimmunerkrankungen (144 RA, 114 SLE, 55 AS, 68 MG) bestimmt. Die Verän- 
derungen der ADA-Aktivität im Serum bei den Patienten wurden analysiert. Die Grenzwertoptimierungskurve (ROC) wurde verwendet, um die Diagnostikleistung der ADA-Aktivitäten im Serum zu beurteilen.

Ergebnisse Im Vergleich zu den gesunden Kontrollen war die tADA-Aktivität im Serum bei den SLE-Patienten deutlich erhöht $(P<0,001)$, während die tADA-Aktivität im Serum bei den Patienten mit RA, AS und MG unverändert blieb $(P>0,05)$. Darüber hinaus wurden in unseren Ergebnissen gezeigt, dass es keine signifikanten Veränderungen bei den ADA1- und ADA2Aktivitäten der RA-, AS- und MG-Patienten gaben, während die ADA2-Aktivität im Serum bei den SLE-Patienten deutlich erhöht wurde. Die ROC-Analyse zeigte, tADA-Aktivität (mit 79,8\% iger Spezifität und 74,6\%iger Sensitivität; LR: 3.693) und ADA2Aktivität (mit 75,4\% iger Spezifität und 78,1\%iger Sensitivität; LR: 3.175) sind unwahrscheinlich bei der diagnose SLE. Außerdem gab es eine positive Korrelation zwischen tADA-Aktivität sowie SLE-Krankheitsaktivität $(r=0,303, P=0,108)$. die SerumtADA-Aktivität bei SLE-Patienten mit Arthritis höher war als bei Patienten ohne Arthritis ( $P=0.005)$

Konklusion Diese Ergebnisse deuten darauf hin, dass die tADA- sowie ADA2-Aktivität im Serum eine wichtige Rolle bei der SLE-Progression spielen könnte

\section{Introduction}

Adenosine deaminase (ADA, EC 3.5.4.4) is a ubiquitously expressed metabolic enzyme, and catalyzes the irreversible deamination of adenosine and deoxyadenosine [1]. Several lines of evidence have identified that high concentration of adenosine in microenvironment acts as a natural brake on immune cell functions, and limits hyperergic inflammatory responses [2,3]. Thus, as the adenosine degrading enzyme, ADA plays an important role in human immune system. Accumulating evidence have revealed its critical role in development, maturation and function of the immune system [4]. ADA activity can partly reflect the immunocompetence in human. Firstly, ADA deficiency results in the severe combined immunodeficiency disease in human $[5,6]$. Patients with ADA deficiency are showing marked lymphopenia. The T, B and NK cells are all dysplastic in patients with ADA deficiency [7,8]. On the other hand, hyperactivity of ADA also could be linked with immune imbalance. Several evidence have shown that serum ADA activity changed in autoimmune diseases, such as systemic lupus erythematosus (SLE) and Rheumatoid arthritis (RA) [9-15]. However, the most of studies were based on small numbers of patients. Thus, more studies using larger cohorts of patients with autoimmune diseases are needed.

RA, SLE, Ankylosing spondylitis (AS) and Myasthenia gravis (MG) are the common autoimmune diseases. In the present study, we investigated the activity of ADA and its isoenzymes (ADA1 and ADA2) in serum of patients with these 4 types of autoimmune diseases. The main objective of this study is to evaluate the change of serum ADA activity in patients with different autoimmune diseases.

\section{Method}

\section{Patients and serum}

Four types of autoimmune diseases were included in this study: 144 RA patients and 144 sex \& age matched healthy controls; 114 patients with SLE and 114 sex \& age matched healthy controls; 55 patients with AS and 55 sex \& age matched healthy controls; 68 patients with MG and 68 sex \& age matched healthy controls. Patients with RA, SLE, AS were recruited from the department of rheumatism and immunology, MG patients were recruited from the de- partment of internal neurology. The corresponding sex \& age matched healthy controls were recruited at the same time. Peripheral blood was collected and subjected to $4000 \mathrm{rpm}$ centrifuge for $5 \mathrm{~min}$ at $4{ }^{\circ} \mathrm{C}$ to obtain serum. Serum was stored at $4{ }^{\circ} \mathrm{C}$ and the serum ADA activity was measured within 7 days. Ethical approval was obtained from the ethics committee of Tangdu Hospital, The Fourth Military Medical University.

\section{Serum ADA activity detection assay}

Serum total ADA (tADA) activity was measured with enzymatic method kit (Sichuan Maccura Biotechnology, China), adapted to the automated biochemistry analyzer (Hitachi 7600, Japan). The serum ADA2 activity was measured in the presence of $0.1 \mathrm{mM}$ erythro9-(2-hydroxy-3-nonyl) adenine (EHNA). The serum ADA1 activity was calculated by subtracting the ADA2 activity from tADA activity. The results were expressed as Unit per Liter (U/L). ADA enzyme remain stable for at least 7 days at $4{ }^{\circ} \mathrm{C}$. In this study, ADA activity was measured within 7 days.

\section{Receiver operating characteristic curve analysis}

Receiver operating characteristic (ROC) curve analysis was applied by plotting sensitivity against (1-specificity) for various levels of serum ADA activity. The optimal cutoff value that best distinguished patients with disease from healthy individuals was determined at maximum value for Youdens' index, which was defined as the difference between the true positive rate and the false positive rate [sensivitiy - (1-specificity)]. The overall diagnostic accuracy was estimated based on area under the ROC curve (AUC).

\section{Statistical analysis}

Serum tADA, ADA1 and ADA2 activity were expressed in median and interquartile range (IQR). Wilcoxon's test was used to analyze the variation of serum ADA activity in patients with autoimmune diseases. Spearman rank correlation analysis was used to estimate the correlation between ADA activity and SLE disease activity. The X2 test was used to compare sex difference between RA, SLE, AS and MG patients. A P-value $<0.05$ was used to define statistical significance. All analyses were performed by using R version 3.4.2. 


\section{Results}

\section{There are significant differences in age and sex characteristics between patients with different autoimmune diseases}

Firstly, we analyzed the age and sex characteristics of RA, SLE, AS and MG patients. RA patients are mostly middle-aged and elderly females (Age: 55.5 (IQR: 47-66); Male/Female: 34/110); SLE pa- tients are mostly young and middle-aged females (Age: 35 (IQR: 27-48.75); Male/Female: 13/101); AS patients are mostly young and middle-aged males (Age: 29 (IQR: 22-40.5); Male/Female: 45/10); MG patients are mostly middle-aged persons (Age: 50 (IQR: 30-60.5); Male/Female: 32/36). These results showed that the age and sex characteristics are significantly different between patients with different autoimmune diseases ( $>$ Table $\mathbf{1})$.

- Table 1 The age and sex characteristics of RA, SLE, AS and MG patients.

\begin{tabular}{|c|c|c|c|c|c|c|c|c|c|c|}
\hline \multirow[t]{2}{*}{ Patients } & \multirow{2}{*}{$\begin{array}{l}\text { Male / } \\
\text { Female }\end{array}$} & \multicolumn{4}{|c|}{$P$ value } & \multirow{2}{*}{$\begin{array}{l}\text { Age } \\
\text { Median } \\
\text { (IQR) }\end{array}$} & \multicolumn{4}{|c|}{$P$ value } \\
\hline & & vs $R A$ & vs SLE & vs AS & vs MG & & vs $R A$ & vs SLE & vs AS & vs MG \\
\hline RA & $34 / 110$ & - & 0.012 & 0.000 & 0.001 & $\begin{array}{l}55.5 \\
(47-66)\end{array}$ & - & 0.000 & 0.000 & 0.001 \\
\hline SLE & $13 / 101$ & 0.012 & - & 0.000 & 0.000 & $\begin{array}{l}35 \\
(27-48.75)\end{array}$ & 0.000 & - & 0.013 & 0.001 \\
\hline AS & $45 / 10$ & 0.000 & 0.000 & - & 0.000 & $\begin{array}{l}29 \\
(22-40.5)\end{array}$ & 0.000 & 0.013 & - & 0.000 \\
\hline MG & 32 / 36 & 0.000 & 0.000 & 0.000 & - & $\begin{array}{l}50 \\
(30-60.5)\end{array}$ & 0.001 & 0.001 & 0.000 & - \\
\hline
\end{tabular}
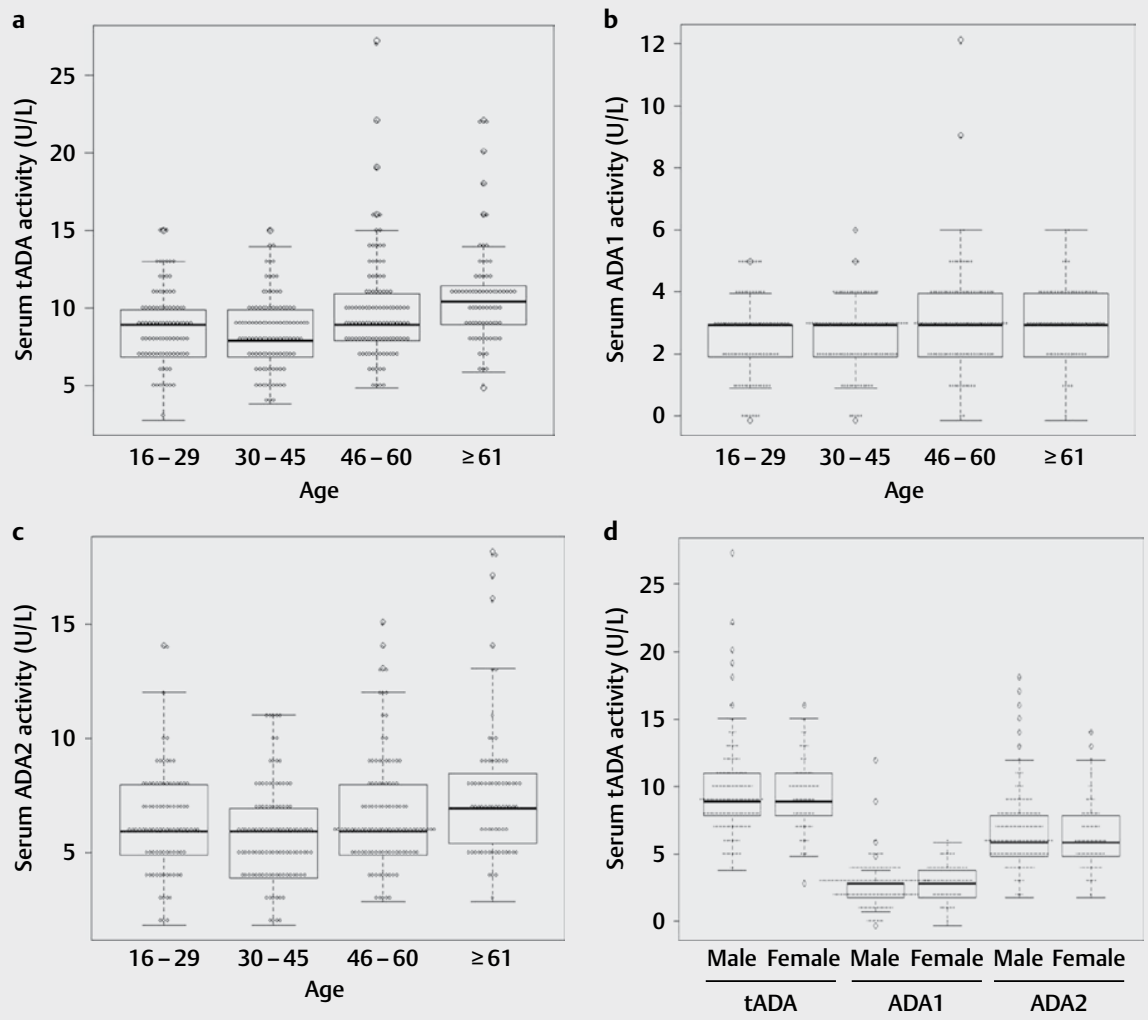

Fig. 1 Box plots showing the serum ADA activity in persons with different age and sex. a, $\mathbf{c}$ The serum tADA and ADA2 activity were increased in persons with age older than $60, \mathbf{b}$ There was no significant difference of serum ADA 1 activity between persons with different age, $\mathbf{d}$ There were no significant differences of serum tADA, ADA1 and ADA2 activity between females and males. 


\section{Serum tADA and ADA2 activity were increased in people older than 60}

To investigate whether the serum ADA activity was different in people with different age, we divided all of healthy subjects $(n=381)$ in this study into 4 groups according to their age: ( $\mathbf{F i g}$. 1a-c). The results showed that there was no significant difference of ADA activity between people with age $\leq 45(P>0.01)$, while the serum tADA and $A D A 2$ activity were both significantly increased in people with age $>60(P<0.01)$. Furthermore, the difference of serum ADA activity between males and females was also analyzed ( $>$ Fig. 1d). The median value of serum tADA activity in males $(n=124)$ and females $(n=257)$ are both 9 (IQR: 8-11) U/L, there was no significant difference of serum tADA activity between males and females $(P=0.344)$, and moreover, there were also no significant differences of serum ADA1 and ADA2 activity between males and females $(P>0.01)$. Taken together, serum ADA activity was independent of sex, while the serum tADA and ADA2 activity were increased in people with age $>60$.

\section{Serum ADA activity pattern in patients with different autoimmune diseases}

\section{Serum ADA activity pattern in RA patients}

The median (IQR) serum tADA, ADA1 and ADA2 activity in 144 RA patients were 10 (IQR: 8-13), 3(IQR: 2-3.25) and 8 (IQR: 5.7510) $\mathrm{U} / \mathrm{L}$, respectively. As the results showed in $>$ Table 2 , compared

- Table 2 Serum ADA activity in RA patients and corresponding healthy controls.

\begin{tabular}{|l|l|l|l|}
\hline & Control & RA & P value \\
\hline Male / Female & $34 / 110$ & $34 / 110$ & - \\
\hline Age (median (IQR)) & $57(46.75-64)$ & $55.5(47-66)$ & 0.899 \\
\hline tADA (median (IQR)) & $10(8-11.25)$ & $10(8-13)$ & 0.125 \\
\hline ADA1 (median (IQR)) & $3(2-4)$ & $3(2-3.25)$ & 0.356 \\
\hline ADA2 (median (IQR)) & $7(5-9)$ & $8(5.75-10)$ & 0.008 \\
\hline
\end{tabular}
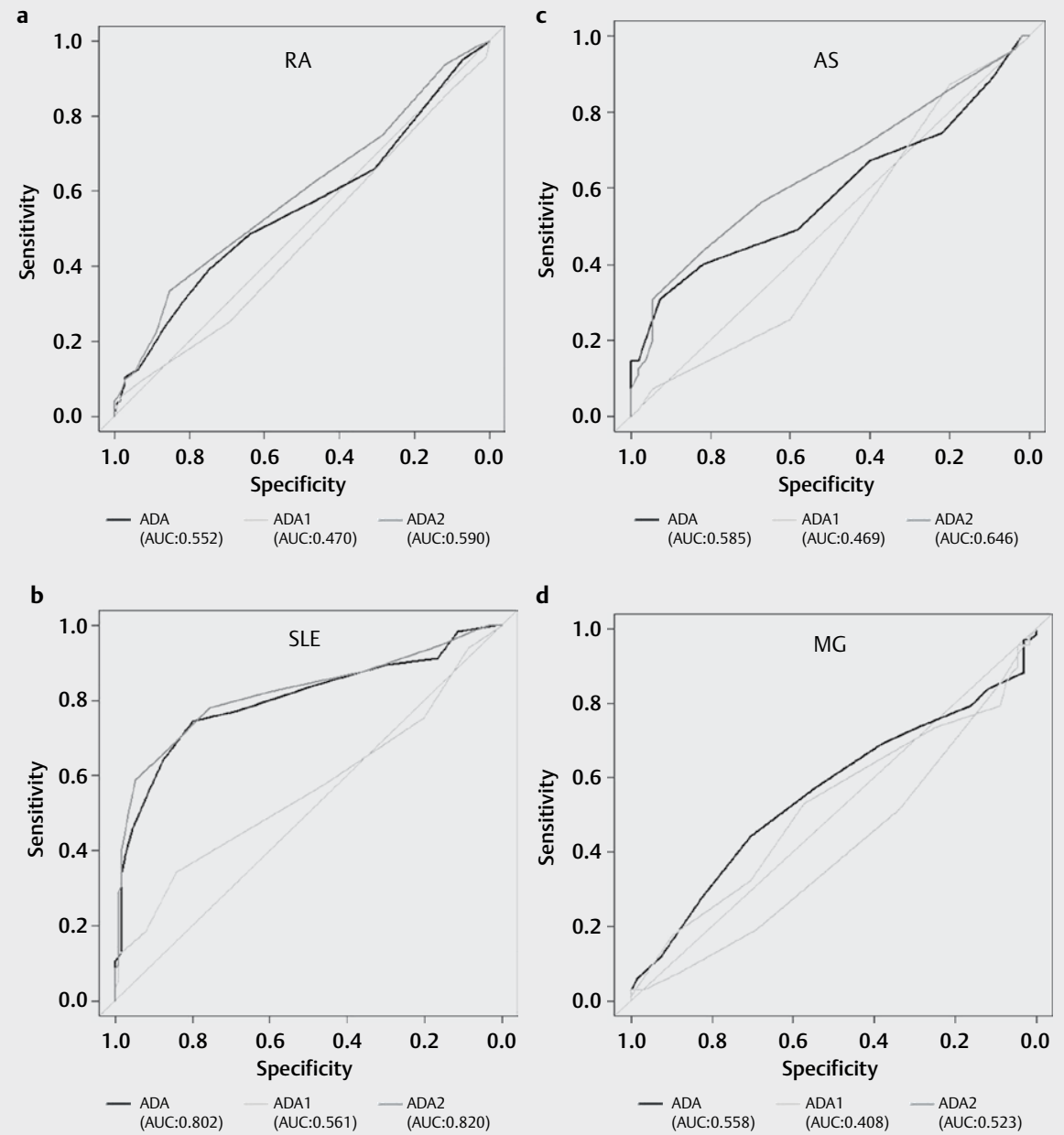

- Fig. 2 ROC curves analysis of serum tADA, ADA1 and ADA2 activity for diagnosing patients with RA, SLE, AS or MG. The results showed that serum ADA activity could not be used to diagnose RA, SLE, AS and MG a, b, $\mathbf{c}, \mathbf{d}$. Serum tADA activity with a cut-off value of $10.5 \mathrm{U} / \mathrm{L}$ yielded the optimal diagnostic performance for SLE patients (79.8 \% specificity and $74.6 \%$ sensitivity; LR: 3.693 ), and serum ADA2 activity with a cut-off value of 7.5 $\mathrm{U} / \mathrm{L}$ yielded the optimal diagnostic performance for SLE patients (75.4 \% specificity and $78.1 \%$ sensitivity; LR: 3.175 ). 
with that in sex- and age- matched healthy controls, the serum tADA and ADA1 activity were not changed in RA patients $(P=0.125$; $P=0.356)$, while serum ADA2 activity were increased slightly $(P=0.008)$. These results showed that serum ADA activity detection could not be used in diagnosing of RA patients ( $\bullet$ Fig. $\mathbf{2 a}$ ).

\section{Serum ADA activity pattern in SLE patients}

The median serum tADA, ADA1 and ADA2 activity in 114 patients with SLE were 13 (IQR: 10.25-17.75), 3(IQR: 2-4) and 11 (IQR: 8-14) U/L, respectively ( $\triangleright$ Table 3). Compared with that in sex- and age- matched healthy controls, the serum tADA and ADA2 activity were significantly increased in SLE patients $(P<0.001 ; P<0.001)$, while there was no significant difference of the serum ADA1 activity between SLE patients and healthy controls $(P=0.104)$. Based on the ROC curve analysis, the optimal cut-off value for tADA and ADA2 activity were $10.5 \mathrm{U} / \mathrm{L}$ and $7.5 \mathrm{U} / \mathrm{L}$ ( $\triangleright$ Fig. 2b). At this level, the specificity and sensitivity of tADA activity were $79.8 \%$ and $74.6 \%$ (LR: 3.693), the specificity and sensitivity of ADA2 were 75.4 and $78.1 \%$ (LR: 3.175 ), respectively. In regard of the low $L R$, the serum ADA activity was unlikely to be used in SLE diagnosing.

\section{Serum ADA activity pattern in AS patients}

The median serum tADA, ADA1 and ADA2 activity in 55 patients with AS were 9 (IQR: 7.5-12), 3 (IQR: 2-3.5) and 7 (IQR: 5-9) U/L, respectively. As the results shown in $>$ Table 4, there were no significant differences of serum $\mathrm{AADA}$ and ADA 1 activity between $\mathrm{AS}$ patients and healthy controls $(P=0.123 ; P=0.571)$, while ADA2 activity in AS patients' serum were increased slightly $(P=0.008)$. Taken together, there data indicated that the serum ADA activity detection would not be helpful in diagnosing of AS patients ( $\mathbf{F i g . ~} \mathbf{2 c}$ ).

- Table 3 Serum ADA activity in SLE patients and corresponding healthy controls.

\begin{tabular}{|l|l|l|l|}
\hline & Control & SLE & P value \\
\hline Male / Female & $13 / 101$ & $13 / 101$ & - \\
\hline Age (median (IQR)) & $35(26.25-46.75)$ & $35(27-48.75)$ & 0.928 \\
\hline tADA (median (IQR)) & $9(7-10)$ & $\begin{array}{l}13(10.25- \\
17.75)\end{array}$ & $<0.001$ \\
\hline ADA1 (median (IQR)) & $3(2-3)$ & $3(2-4)$ & 0.104 \\
\hline ADA2 (median (IQR)) & $6(5-7)$ & $11(8-14)$ & $<0.001$ \\
\hline
\end{tabular}

- Table 4 Serum ADA activity in AS patients and corresponding healthy controls.

\begin{tabular}{|l|l|l|l|}
\hline & Control & AS & P value \\
\hline Male / Female & $45 / 10$ & $45 / 10$ & - \\
\hline Age (median (IQR)) & $30(23.5-41)$ & $29(22-40.5)$ & 0.711 \\
\hline tADA (median(IQR)) & $9(8-10)$ & $9(7.5-12)$ & 0.123 \\
\hline ADA1 (median (IQR)) & $3(2-4)$ & $3(2-3.5)$ & 0.571 \\
\hline ADA2 (median (IQR)) & $6(5-7)$ & $7(5-9)$ & 0.008 \\
\hline
\end{tabular}

\section{Serum ADA activity pattern in MG patients}

The median serum tADA, ADA1 and ADA2 activity in 68 patients with MG were 8 (IQR: 6-11), 3 (IQR: 2-3) and 5 (IQR: 4-8) U/L, respectively. As the results shown in $>$ Table 5 , there were no significant differences of serum tADA, ADA1 and ADA2 activity between MG patients and corresponding healthy controls $(P=0.243$; $P=0.056 ; P=0.64)$. Therefore, serum ADA activity could not be a diagnostic marker for MG patients ( $\triangleright$ Fig. 2 d).

\section{The association between serum ADA activity and manifestation of SLE patients}

Compared with that in healthy controls and patients with the other autoimmune diseases (RA, AS and MG), the serum tADA and ADA2 activity were significantly increased in SLE patients ( $\triangleright$ Fig. $3 a-c$ ). We further analyzed the correlation between serum ADA activity and SLE disease activity by using spearman's correlation analysis. SLE disease activity was assessed by using the Systemic Lupus Erythematosus Disease Activity Index 2000 (SLEDAI). The result showed that there was a positive correlation between tADA activity and SLEDAI $(r=0.303, P=0.010)$. Furthermore, we analyzed the association between serum ADA activity and manifestation of SLE patients ( $\triangleright$ Table 6). The results showed that the positive rate of multiple manifestations such as low complement (73.91\%), rash (56.52\%), anti-dsDNA antibodies (53.62\%), arthritis (39.13\%) and proteinuria $(31.88 \%)$ were lower than the positive rate of increased serum tADA (74.6\%) in SLE patients. Notably, the serum tADA activity in SLE patients with arthritis were higher than that in patients without arthritis ( $\triangleright$ Table $6, P=0.005$ ), which suggested that tADA activity might be related to subtype of SLE with arthritis.

\section{Discussion}

ADA plays an important role in growth and differentiation of lymphocytes and macrophages. ADA activity is elevated during T cell response, and is considered as a non-specific marker of $T$ lymphocyte mediated immunoresponse [16]. Thus ADA activity may change in patients with abnormal immunity. In the present study, we aimed to evaluate the change of ADA and its isoenzymes (ADA1 and ADA2) activity in patients with different autoimmune diseases. To the end, we investigated the serum ADA activity in four types of

- Table 5 Serum ADA activity in MG patients and corresponding healthy controls.

\begin{tabular}{|l|l|l|l|}
\hline & Control & MG & P value \\
\hline Male / Female & $32 / 36$ & $32 / 36$ & - \\
\hline $\begin{array}{l}\text { Age } \\
\text { (median (IQR)) }\end{array}$ & $47.5(33.75-58.25)$ & $50(30-60.5)$ & 0.850 \\
\hline $\begin{array}{l}\text { tADA } \\
\text { (median (IQR)) }\end{array}$ & $9(7-11)$ & $8(6-11)$ & 0.243 \\
\hline $\begin{array}{l}\text { ADA1 } \\
\text { (median (IQR)) }\end{array}$ & $3(2-4)$ & $3(2-3)$ & 0.056 \\
\hline $\begin{array}{l}\text { ADA2 } \\
\text { (median (IQR)) }\end{array}$ & $6(4-7.25)$ & $5(4-8)$ & 0.64 \\
\hline
\end{tabular}



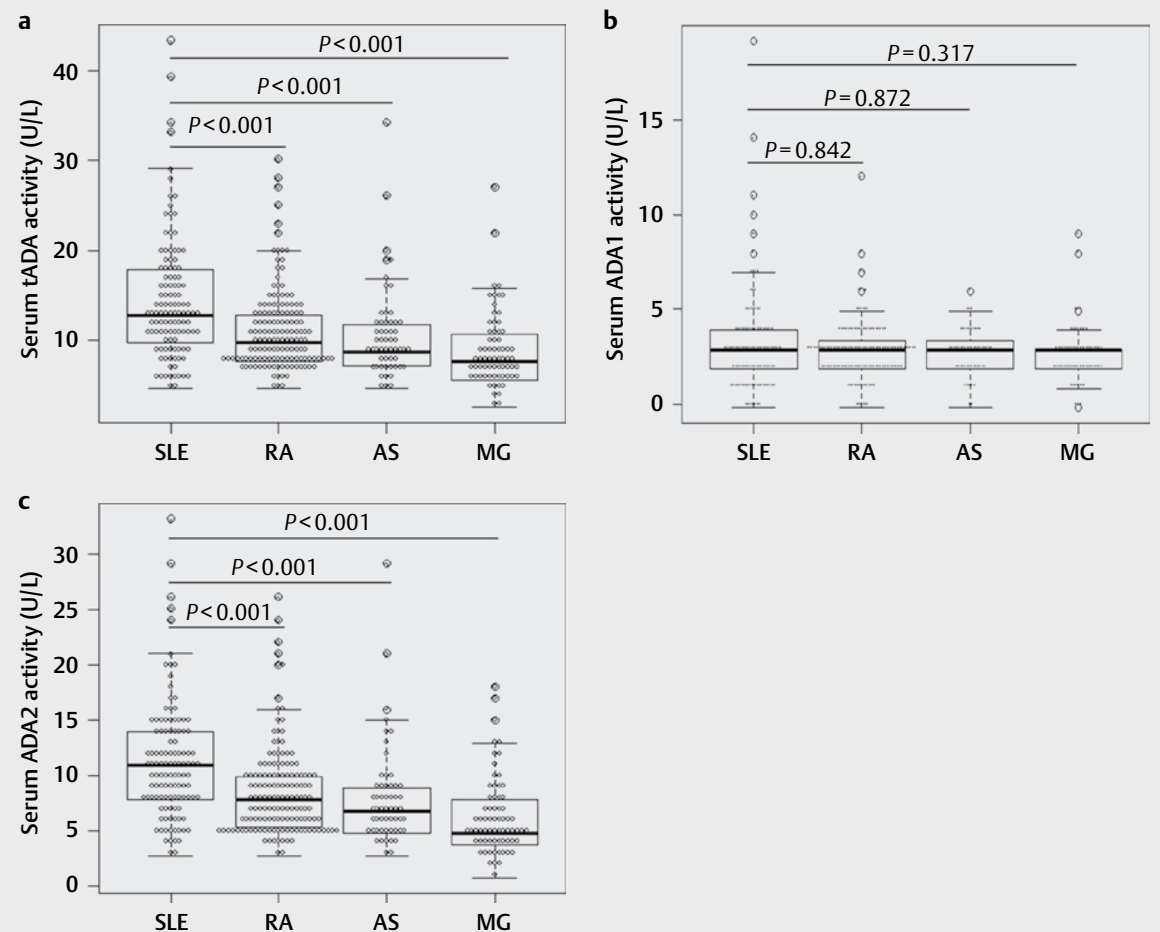

- Fig. 3 The difference of serum ADA activity between SLE and other autoimmune diseases: RA, AS and MG patients. a, c Serum tADA and ADA2 activity in SLE patients were higher than that in RA, AS and MG patients. b There was no difference of serum ADA1 activity between RA, AS, MG and SLE patients.

- Table 6 The association between serum tADA activity and manifestations of SLE patients.

\begin{tabular}{|l|l|l|l|l|}
\hline Manifestations & Positive rate (\%) & $\begin{array}{l}\text { tADA activity in } \\
\text { positive patients (U/L) }\end{array}$ & $\begin{array}{l}\text { tADA activity in } \\
\text { negative patients (U/L) }\end{array}$ \\
\hline Low complement & & $15(12-20)$ & $13.5(10.25-16.75)$ \\
\hline Rash & 73.91 & $15(13-22)$ & $13.5(9-17.75)$ & 0.137 \\
\hline Anti-dsDNA antibodies & 56.52 & $16(13-20)$ & $14(11-17.25)$ & 0.091 \\
\hline Arthritis & 53.62 & $17(14-22)$ & $13(10.25-16)$ & 0.005 \\
\hline Proteinuria & 39.13 & $14.5(13-17.75)$ & $15(12-20)$ & 0.791 \\
\hline Fever & 31.88 & $19(12-22)$ & $15(11.5-18)$ & 0.247 \\
\hline Leukopenia & 26.08 & $15(12-20)$ & $15(11.5-19)$ & 0.758 \\
\hline Alopecia & 26.08 & $14(12-22)$ & $15(12-19)$ & 0.660 \\
\hline Thrombocytopenia & 24.63 & $16(13-21)$ & $14.5(12-18)$ & 0.250 \\
\hline Hematuria & 21.74 & $14(12-21)$ & $15(12-19)$ & 0.943 \\
\hline Myositis & 17.39 & $16(11.5-21.5)$ & $15(12-19.5)$ & 0.765 \\
\hline Pyuria & 10.14 & $16(12.5-22)$ & $14.5(12-19.75)$ \\
\hline Mucosal ulcers & 10.14 & $15(13.5-21)$ & $15(12-20)$ \\
\hline Pleurisy & 10.14 & $14(12.25-18)$ & 0.661 \\
\hline
\end{tabular}

autoimmune diseases (RA, SLE, AS and MG). The findings are summarized as follows: 1) Serum tADA and ADA2 activity were significantly increased in SLE patients as compared with that in healthy controls, while there were no differences of ADA activity between patients with RA, AS, MG and healthy controls. 2) Base on ROC curve analysis, serum tADA activity $(79.8 \%$ specificity and $74.6 \%$ 
sensitivity; LR: 3.693 ) and ADA2 activity (75.4\% specificity and $78.1 \%$ sensitivity; LR: 3.175 ) are unlikely to be used in diagnosing SLE.3) Serum tADA activity was positive correlated with SLE disease activity $(r=0.303, P=0.011)$. The serum tADA activity might be related to lupus arthritis.

In this study, four common autoimmune diseases and the corresponding sex- and age- matched healthy controls were recruited. The sex and age characteristics of RA, SLE, AS and MG patients are different. RA and SLE patients are mostly females, AS patients are mostly males, while the morbidity of MG in female and males is about the same. The median age of RA patients is significantly older than SLE, AS and MG patients. By dividing healthy subjects into different groups according to their sex or age, our results showed that the serum ADA activity were independent of sex, while were increased in people older than 60 .

Our results showed that there were no significant differences of serum ADA activity between patients with RA, AS, MG and healthy controls. However, several studies previously reported that serum ADA activity was increased in RA and MG patients. Sari RA et al. and Vinapamula KS et al., have shown the increased serum ADA activity in 37 and 46 RA patients, respectively $[9,17]$. Chiba $S$ et al., have reported higher serum ADA activity in $31 \mathrm{MG}$ patients than that in controls $[18,19]$. These conflict results indicated that the change of serum ADA activity between ethnicities might be different. Notably, the small number of samples included in above studies may cause inaccuracy. Therefore, further studies in larger cohorts are needed.

The serum tADA and ADA2 activity in SLE patients were significantly increased compared with that in healthy controls. This finding about serum tADA activity in SLE is consistent with our previous reports [20]. And moreover, serum tADA and ADA2 activity in SLE patients were significantly higher than that in patients with the other autoimmune diseases, which means that serum tADA and ADA2 activity detection could be helpful in distinguish SLE patients from other autoimmune diseases. Notably, there was a positive correlation between tADA activity and SLE disease activity, which suggested that tADA activity might be helpful in monitoring the disease activity. Several studies have reported that interferon level (such as IFN-1 $\alpha$, IFN- $\alpha$ and IFN- $\lambda 3$ ) were increased in SLE patients [21-23], which suggested the change of interferon signature were involved in SLE development. We will investigate the potential association between ADA activity and interferon in SLE patients in further study. In conclusion, our data suggested that increased ADA activity might play an important role in SLE progression, especially in lupus arthritis.

\section{Acknowledgment}

This study is supported by the National Natural Science Foundation of China (no. 81702732). All authors have read and approved the final version for submission.

\section{Conflict of Interest}

The authors declare that there is no conflict of interests regarding the publication of this paper.
References

[1] Antonioli L, Colucci R, La Motta C et al. Adenosine deaminase in the modulation of immune system and its potential as a novel target for treatment of inflammatory disorders. Current drug targets 2012; 13 : 842-862

[2] Burnstock G, Boeynaems JM. Purinergic signalling and immune cells. Purinergic signalling 2014; 10: 529-564

[3] Ohta A, Sitkovsky M. Extracellular adenosine-mediated modulation of regulatory T cells. Frontiers in immunology 2014; 5: 304

[4] Flinn AM, Gennery AR. Adenosine deaminase deficiency: a review. Journal of animal physiology and animal nutrition 2018; 13: 65

[5] Bradford KL, Moretti FA, Carbonaro-Sarracino DA et al. Adenosine Deaminase (ADA)-Deficient Severe Combined Immune Deficiency (SCID): Molecular Pathogenesis and Clinical Manifestations. Journal of clinical immunology 2017; 37: 626-637

[6] Whitmore KV, Gaspar HB. Adenosine Deaminase Deficiency - More Than Just an Immunodeficiency. Frontiers in immunology 2016; 7: 314

[7] Blackburn MR, Aldrich M, Volmer JB et al. The use of enzyme therapy to regulate the metabolic and phenotypic consequences of adenosine deaminase deficiency in mice. Differential impact on pulmonary and immunologic abnormalities. The Journal of biological chemistry 2000; 275: 32114-32121

[8] Blackburn MR, Kellems RE. Adenosine deaminase deficiency: metabolic basis of immune deficiency and pulmonary inflammation. Advances in immunology 2005; 86: 1-41

[9] Vinapamula KS, Pemmaraju SV, Bhattaram SK et al. Serum Adenosine Deaminase as Inflammatory Marker in Rheumatoid Arthritis. Journal of clinical and diagnostic research : JCDR 2015; 9: Bc08 10

[10] Saghiri R, Ghashghai N, Movaseghi S et al. Serum adenosine deaminase activity in patients with systemic lupus erythematosus: a study based on ADA1 and ADA2 isoenzymes pattern. Rheumatology international 2012; 32: 1633-1638

[11] Hitoglou S, Hatzistilianou M, Gougoustamou D et al. Adenosine deaminase activity and its isoenzyme pattern in patients with juvenile rheumatoid arthritis and systemic lupus erythematosus. Clinical rheumatology 2001; 20: 411-416

[12] Nalesnik M, Nikolic JM, Jandric S. Adenosine deaminase and C-reactive protein in diagnosing and monitoring of rheumatoid arthritis. Medicinski glasnik: official publication of the Medical Association of Zenica-Doboj Canton, Bosnia and Herzegovina 2011; 8: 163-168

[13] Demir G, Borman P, Ayhan F et al. Serum Adenosine Deaminase Level is High But Not Related with Disease Activity Parameters in Patients with Rheumatoid Arthritis. The open rheumatology journal 2014; 8: 24-28

[14] Zamani B, Jamali R, Jamali A. Serum adenosine deaminase may predict disease activity in rheumatoid arthritis. Rheumatology international 2012; 32: 1967-1975

[15] Stancikova M, Lukac ], Istok R et al. Serum adenosine deaminase activity and its isoenzyme pattern in patients with systemic lupus erythematosus. Clinical and experimental rheumatology 1998; 16: 583-586

[16] Cristalli G, Costanzi S, Lambertucci C et al. Adenosine deaminase: functional implications and different classes of inhibitors. Medicinal research reviews 2001; 21: 105-128

[17] Sari RA, Taysi S, Yilmaz O et al. Correlation of serum levels of adenosine deaminase activity and its isoenzymes with disease activity in rheumatoid arthritis. Clinical and experimental rheumatology 2003; 21: $87-90$

[18] Chiba S, Saitoh M, Kashiwagi M et al. Isozyme analysis of the high serum adenosine deaminase activity in patients with myasthenia gravis. Internal medicine (Tokyo, Japan) 1995; 34: 81-84 
[19] Chiba S, Matsumoto H, Motoi Y et al. High serum adenosine deaminase activity and its correlation with lymphocyte subsets in myasthenia gravis. Journal of the neurological sciences 1990; 100: 174-177

[20] Gao ZW, Zhao GH, Zhang Z et al. Serum adenosine deaminase activity is increased in systemic lupus erythematosus patients and correlated with disease activity. Immunologic research 2018; 66: 299-304

[21] Mathian A, Mouries-Martin S, Dorgham K. Monitoring Disease Activity in Systemic Lupus Erythematosus With Single-Molecule Array Digital Enzyme-Linked Immunosorbent Assay Quantification of Serum Interferon-alpha. 2019; 71: 756-765
[22] Fernandez Matilla M, Grau Garcia E, Fernandez-Llanio Comella N et al. Increased interferon-1alpha, interleukin-10 and BLyS concentrations as clinical activity biomarkers in systemic lupus erythematosus. Medicina clinica 2019; pii S0025-S7753 (19)30024-7

[23] Amoura Z, Chen JY, Wang CM et al. Interferon-lambda3/4 genetic variants and interferon-lambda 3 serum levels are biomarkers of lupus nephritis and disease activity in Taiwanese. Arthritis \& rheumatology 2018; 20: 193 